\title{
Stroke and atrial fibrillation in sick sinus syndrome
}

After the first electrocardiographic description of sinus node disease in 1923 by Wenckebach, it took about 50 years until the clinical entity of sinus node disorder, including its electrocardiographic parameters and clinical symptoms, was established. Currently between $40 \%$ and $50 \%$ of patients in Europe and the United States that receive a pacemaker have sinus node disease as the underlying rhythm disorder. Of those, $20 \%$ to $30 \%$ have had symptoms of paroxysmal or intermittent atrial fibrillation as part of the sinus node disorder. This entity is also called brady-tachy syndrome.

This syndrome, which was first described in 1968, summarises various manifestations of sinoatrial rhythm disorders:

- serious, persistent, otherwise inexplicable sinus bradycardias that often represent the initial symptom;

- sinus arrest with replacement of the sinus rhythm by heterotopic atrial or nodal rhythms, or long periods of cardiac arrest in situations in which the latter fail;

- paroxysmal or chronic atrial fibrillation due to sinus arrest;

- an inadequate sinus node activity post-cardioversion of atrial fibrillation;

- sinoatrial exit blocks unrelated to medical treatments;

- inadequate heart rate increase during incremental exercise.

The activity of the sinus node, which consists of four different cell types capable of engendering sinus node automaticity, is conducted to the atrioventricular node via three physiological pathways (James, Wenckebach, and Thorel). The content of sinus node cells involved in normal impulse formation has been shown to diminish with advanced age, but without causing serious damage to the function of the sinus node unless more than $90 \%$ of the content is destroyed. Additional mechanisms forming the anatomical basis of sinus node syndrome are destruction of the sinus node itself or of nodoatrial areas resulting from-for example, ischaemia, inflammatory or degenerative changes of the nerves and ganglia surrounding the sinus node, and pathological changes in the atrial myocardial wall such as fibrosis or fatty infiltration, all of which lead to dispersion of refractoriness and to a decrease in conduction velocity. Consequently, heterotopic atrial or nodal rhythms emerge depriving an otherwise normal heart of its active atrial contraction during the cardiac cycle. A disarrayed and less forceful atrial movement, especially with atrial fibrillation, leads to blood stasis within the atria and their appendages, forming the basis for thromboembolism.

Though early studies by Shaw and Kekwick ${ }^{1}$ and by our group ${ }^{2}$ showed that the survival rate of patients with sick sinus syndrome was similar to that of age and sex matched controls, complications and morbidity associated with sinus node disorder, such as stroke, have become of greater interest in recent years.

\section{Complications associated with atrial fibrillation}

As a result of increasing interest in atrial fibrillation and its complications, both atrial fibrillation and sinus node disorder have recently been studied intensively. The Framingham Study ${ }^{34}$ showed that $15-20 \%$ of stroke patients have atrial fibrillation, that $45-70 \%$ of strokes in patients with atrial fibrillation are caused by embolic events, and that the risk of stroke is $2-7 \%$ per year. In addition, it has been shown that $2 \%$ of patients with intermittent or paroxysmal atrial fibrillation have strokes each year and that a transition from paroxysmal to chronic atrial fibrillation occurs in about one third of these patients at a median of 34 months. ${ }^{5}$ This transition from paroxysmal to chronic atrial fibrillation significantly increases strokes to about $5 \%$ per year $(P<0.05))^{34}$

Recent trials on anticoagulation, especially low dose warfarin, in patients with atrial fibrillation have focused attention on the morbidity and mortality associated with atrial fibrillation. Strokes were reduced from $3-7 \cdot 4 \%$ annually without treatment to $0 \cdot 4-2 \cdot 6 \%$ with treatment, a considerable therapeutic benefit.

Retrospective trials linking pacing and sick sinus syndrome with complications

The first study that showed that the mode of pacing in patients with sick sinus syndrome had an effect on mortality and morbidity was that of Rosenqvist et al. ${ }^{6}$ In this study, 168 patients with sick sinus syndrome treated at two hospitals in Sweden received either a VVI system at one hospital or an AAIR pacemaker at the other. Both groups had similar baseline clinical characteristics, characteristics of sinus node disorder, or prior intermittent atrial tachyarrhythmias. At a mean follow up of four years, $4 \%$ of the patients with an AAIR pacemaker developed chronic atrial fibrillation compared with $39 \%$ of patients with the VVI system. There was also a slight significant difference in the development of congestive heart failure ( $7 \% v 23 \%)$ and the incidence of stroke (4.5\% $v 13 \%)$. Whatever type of pacemaker was implanted, the incidence of cerebrovascular disease and stroke was $23 \%$ in the patients who developed chronic atrial fibrillation and $6 \%$ in patients who remained in sinus rhythm.

This first study triggered similar investigations that analysed the dataset from larger patient populations. Hesselson et al analysed data from 950 pacemaker implants over a period of seven years and found a significant increase in the development of chronic atrial fibrillation in patients in whom a VVI pacemaker was implanted 
compared with patients in whom a physiological DDD or VVD system was implanted. ${ }^{7}$ The incidence of atrial fibrillation was highest in patients with sick sinus syndrome and a prior history of atrial fibrillation. Concomitantly, patients older than 70 years had an extraordinarily high likelihood of developing atrial fibrillation if they received a VVI pacemaker for treatment of sick sinus syndrome. This is particularly important as this group may not be as easy or safe to treat with anticoagulation.

A study from the Cleveland Clinic ${ }^{8}$ analysed the outcome in 507 patients with sick sinus syndrome with respect to two end points: development of chronic atrial fibrillation and the incidence of stroke. The incidence of atrial fibrillation was $7 \%$ at one year, $16 \%$ at five years, and $28 \%$ at 10 years; the incidence of stroke was $30 \%$ at 10 years. Independent risk factors for chronic atrial fibrillation were a history of paroxysmal atrial fibrillation (hazard ratio $16 \cdot 8$ ), ventricular pacing mode (hazard ratio 1.98 ), age, and heart valve disease. Independent predictors of stroke were ventricular pacing mode (hazard ratio $2 \cdot 6$ ) and a history of paroxysmal atrial fibrillation (hazard ratio $2 \cdot 8$ ).

The mortality and stroke in patients with sick sinus syndrome with respect to pacing system was also highlighted in a recent study by Santini et al. ${ }^{9}$ They not only found an incidence of $47 \%$ of chronic atrial fibrillation in patients treated with VVI pacemakers, but also a significantly increased mortality of $8 \% v 2.5 \%$ from stroke in patients with VVI compared with patients with AAI or DDD pacemakers. Similar to the paper from Hesselson et al that showed that patients older than 70 years have a significantly higher risk of experiencing a fatal stroke, ${ }^{7}$ Santini $e t$ al observed that there was a stroke mortality of $16 \% v 3 \%$ in patients with a VVI compared with a physiological system. ${ }^{9}$

The abundance of retrospective literature regarding pacing mode, development of chronic atrial fibrillation, and the incidence of thromboembolism has been amplified by recent summaries compiled by Rosenqvist et $a l^{6}$ Sutton and Kenny, ${ }^{10}$ and Camm and Katritsis ${ }^{11}$ who compared the data from different studies. These reports showed that the annual incidence of atrial fibrillation among all the studies was $5-15 \%$ for VVI and $1-3 \%$ for AAI. In the analysis by Camm and Katritsis, ${ }^{11}$ the incidence of thromboembolism in sick sinus syndrome treated with VVI pacing ranged from $10 \%$ to as high as $48 \%$.

\section{Prospective studies}

There are currently only two prospective trials investigating the outcome depending on pacing mode in sick sinus syndrome and atrial fibrillation. The trial by Andersen et $a^{12}$ analysed 225 consecutive patients that were followed up for five years. Patients with sick sinus syndrome had been randomised to either atrial or ventricular pacing. During follow up, the frequency of atrial fibrillation was higher in the ventricular paced group; thromboembolic events occurred in 20 patients $(17 \%)$ in the ventricular group versus six $(5 \%)$ with atrial pacing $(P<0.008)$. Twenty five patients $(22 \%)$ died in the ventricular group compared with $21(19 \%)$ in the atrial group. While this study found a significant difference regarding occurrence of atrial fibrillation and stroke, the two pacing mode groups exhibited no significant difference in mortality.

Similar results were reported in a recent study by Lamas et al. ${ }^{13}$ In their preliminary PASE study (PAcemaker Selection in the Elderly), a multicentre, prospective, randomised trial, 403 patients with sick sinus syndrome were randomised to either VVI or DDDR pacing. At one year follow up, mortality was $12 \%$ in the VVIR group versus $6 \%$ in the DDDR group, incidence of stroke was $4 \%$ versus $2 \%$, and combined events were $19 \%$ and $10 \%$, respectively. For patients with AV block, the death rate was $10 \% v 9 \%$, stroke $2 \% v 0 \%$, and combined events $19 \% v 13 \%$. While none of these events reached the level of significance, there was an unambiguous trend towards higher death rate and higher stroke occurrence in patients with sick sinus syndrome in VVIR. For patients with AV block virtually no difference with respect to mortality was evident; however, strokes and combined events occurred more frequently in the VVIR paced group.

\section{Limitations and criticisms}

A recent paper ${ }^{14}$ expresses considerable criticism regarding the analysis of retrospective data. In this analysis of more than 38000 patient files from the Medicare/ Medicaid database, a significant selection bias was found regarding the type of pacemaker. While it is true that in clinical practice it is more likely that patients who are older or sicker, or have compromised myocardial function, are more likely to receive a single chamber than a dual chamber pacemaker, the point made in these retrospective and prospective studies that a higher incidence of stroke and death from stroke are observed among patients paced VVI for sick sinus syndrome cannot be discarded. Currently, the general practice of pacing differs considerably between Europe and the United States. In Europe the use of physiological pacemakers ranges between $20 \%$ and $30 \%$, in the United States the range is $50 \%$ to $70 \%$. Therefore, it is not surprising that in the United States some criticism on the use of high end products has been expressed concerning their use in patients for whom physiological pacing might have only marginal benefit. The hypothetical case that may best highlight this is the patient with sinus node disorder and intermittent atrial fibrillation who receives a sophisticated DDDR pacemaker. The patient develops chronic atrial fibrillation a few days after implant and spontaneous healing of the sinus node syndrome without the need of a pacemaker for the rest of his or her life as AV conduction is not compromised. In this patient, the implantation of a DDDR device is of little benefit.

At present, there is a lack of convincing prospective studies that indicate which patient with sick sinus syndrome would best be treated with which mode of pacing. In addition to conventional atrial and ventricular pacing, new forms of treatment have been developed over the past few years, for example, the dual site pacing described by the Saksena group ${ }^{15}$ and by Claude Daubert et al. ${ }^{16}$ Both have shown that in a certain type of sinus node disorder with paroxysmal atrial fibrillation, the stimulation of the atrium from two sides may be beneficial in reducing the recurrence of atrial fibrillation. Other new forms of treatment include the electrical ablation of the atrium to prevent atrial fibrillation in the form of an electrical corridor similar to the maze operation, and the implantation of an atrial defibrillator that shocks patients back to sinus rhythm; however, these are all under clinical investigation.

In contrast, medical treatment does not appear to confer relevant benefit with respect to long term stabilisation of the disease as, although the occurrence of atrial fibrillation can be prevented in a meaningful percentage of cases at least for some time, the natural course of the syndrome and its manifestations have to be taken into consideration.

\section{Current selection criteria}

Though new forms of treatment are on the verge of clinical investigation, current modes of treatment should be based 
on established clinical wisdom. Surely, a patient with sick sinus syndrome, constant sinus bradycardia, and retrograde atrial-ventricular conduction will presumably derive little benefit from the implantation of a pure VVI pacemaker, while a patient with sick sinus syndrome and infrequent, intermittent sinoatrial block that clinically manifests as intermittent syncope may be provided with a VVI pacemaker programmed to a low hysteresis rate. In patients in whom intermittent atrial fibrillation is present, the use of atrial pacing can help to establish antiarrhythmic treatment. If a patient has a long history of atrial fibrillation, considerable enlargement of the atria, and coronary artery disease with fibrosis in the atria, the long term chances of maintaining sinus rhythm may be low; this patient may fare better long term with low dose warfarin and a ventricular pacing system.

Atrial pacing should be the norm in patients with sick sinus syndrome, even in the elderly, as in this group the incidence of atrial fibrillation with VVI pacing is higher, the incidence of thromboembolic complications with atrial fibrillation is higher, and the difficulties associated with warfarin therapy are so high that the benefit compared with aspirin is less than clear. However, there are economic issues that argue against the routine use of dual chamber pacemakers. These are usually selected because of lack of confidence in atrioventricular conduction in these patients. However, this is not supported by clinical data, for example in the Danish study ${ }^{12}$ the need for upgrading from AAI to DDD was very low (a Wenckebach point was measured at implant). Perhaps we should consider more systematic use of AAI rather than dual chamber pacing in sick sinus syndrome.

In addition to currently available data, ${ }^{17}$ the outcome of randomised prospective studies, such as PASE and MOSSST (MOdes Switch Selection STudy), which will include more than 2000 patients with sick sinus syndrome, will shed more light on the benefits and risks of individual pacing therapies for sick sinus syndrome in light of additional clinical variables. Based on the data we received from the anticoagulation studies, the use of low dose warfarin currently provides a powerful means of reducing stroke and mortality in patients with paroxysmal and chronic atrial fibrillation. ${ }^{18}$ With the forces of our changes in reimbursement and economic constrictions, the demonstration of a clinical benefit and cost effectiveness will be needed for justifying a widespread use of more than $50 \%$ dual chamber pacemakers. Perhaps the simple AAI pacemaker favoured in Europe (particularly in Scandinavia with excellent results) but less so in other countries (presumably due to liability reasons and assumed uncertainties regarding the development of atrioventricular conduction disturbances in the further course of the syndrome), may be a cost effective solution to some of the problems without anticipating the results of the prospective, randomised multicentre studies that will be completed between 1998 and 2000

ECKHARD ALT GÜNTER LEHMANN

Department of Cardiology

I. Medizinische Klinik,

Klinikum rechts der Isar

Ismaninger Str. 22, D-81675 München, Germany,

e-mail:alt@med1.med.tu-muenchen.de

1 Shaw DB, Kekwick CA. Potential candidates for pacemakers: survey of heart block and sinoatrial disorder (sick sinus syndrome). Br Heart $\mathcal{F}$ 1978;40:99-105.

2 Alt E, Völker R, Wirtzfeld A, Ulm K. Survival and follow up after pacemaker implantation: a comparison of patients with sick sinus syndrome, complete heart block and atrial fibrillation. $P A C E$ 1985;8:849-55.

3 Kannel WB, Abbott RD, Savage DD, McNamara PM. Epidemiologic features of chronic atrial fibrillation: the Framingham Study. $N$ Engl $₹ \mathrm{Med}$ 1982;306:1018-22.

4 Wolf PA, Abbott RD, Kannel WB. Atrial fibrillation as an independent risk factor for stroke: the Framingham Study. Stroke 1991;22:983-8.

5 Petersen P Anticoagulant therapy for atrial fibrillation. In. Falk RH, Podrid PJ, eds. Atrial fibrillation. Mechanisms and management. New York: Raven Press, 1992:307-19.

6 Rosenqvist M, Brandt J, Schüller H. Long-term pacing in sinus node disease: effects of stimulation mode on cardiovascular morbidity and morease: effects of stimulation mode

7 Hesselson AB, Parsonnet V, Bernstein AD, Bonavita G. Deleterious effects of long-term single-chamber ventricular pacing in patients with sick sinus syndrome: the hidden benefits of dual-chamber pacing. $f$ Am Coll Cardio $1992 ; 19: 1542-9$

8 Sgarbossa EB, Pinski SL, Maloney JD, Simmons TW, Wilkoff BL, Castle $\mathrm{LW}$, et al. Chronic atrial fibrillation and stroke in paced patients with sick sinus syndrome. Circulation 1993;88:1045-53.

9 Santini M, Alexidou G, Ansalone G, Cacciatore G, Cini R, Turitto G Relation of prognosis in sick sinus syndrome to age, conduction defects and modes of permanent cardiac pacing. Am $f$ Cardiol 1990;65:729-35.

10 Sutton R, Kenny RA. The natural history of sick sinus syndrome. $P A C E$ 1986;9:1110-14.

11 Camm AJ, Katritsis D. Ventricular pacing for sick sinus syndrome-a risky business? PACE 1990;13:695-9.

12 Andersen HR, Thuesen L, Bagger JP, Vesterlund T, Thomsen PEB. Prospective randomised trial of atrial versus ventricular pacing in sicksinus syndrome. Lancet 1994;344:1523-8.

13 Lamas GA, Stambler B, Mittelman R, Wilkoff B, Keim S, Brinker J, et al. Clinical events following DDDR versus VVIR pacing: results of a prospective trial [abstract]. $P A C E$ 1996;19:619.

14 Lamas GA, Pashos CL Normand SLT, McNeil B. Permanent pacemake selection and subsequent survival in elderly Medicare pacemaker recipiselection and subsequent survival

15 Prakash A, Hill M, Krol RB, Lewis C, Matthew P, Munsif AN, et al. Longterm trial atrial pacing for prevention of atrial fibrillation: selection of

16 Daubert C, Mabo P, Gras D, Leclercq C, Lelièvre T. Physiological cardiac pacing: an individual objective. In: Aubert AE, Ector H, Stroobandt R, eds. Cardiac pacing and electrophysiology. Boston: Kluwer Academic Publishers 1994:227-50.

17 Kamalvand K, Tan K, Holt P, Bucknall C, Sulke N. Comparison of dua chamber pacing and single chamber ventricular pacing on the incidence of atrial tachyarrhythmias [abstract]. PACE 1996;19:619.

18 Boston Area Anticoagulation Trial for Atrial Fibrillation Investigators. The effect of low-dose warfarin on the risk of stroke in patients with nonrheumatic atrial fibrillation. $N$ Engl $\mathcal{F}$ Med 1990;323:1505-11. 